\title{
Implicações Farmacogenéticas de Polimorfismos da eNOS para Drogas de Ação Cardiovascular
}

\author{
Pharmacogenetic Implications of the eNOS Polymorphisms for Cardiovascular Action Drugs \\ Pâmela Souza Silva', Riccardo Lacchini', Valéria de Aguiar Gomes ${ }^{1}$, José Eduardo Tanus-Santos² \\ Faculdade de Ciências Médicas - Departamento de Farmacologia - Universidade Estadual de Campinas (UNICAMP)' ${ }^{1}$, Campinas, SP; Faculdade \\ de Medicina de Ribeirão Preto - Departamento de Farmacologia - Universidade de São Paulo (USP)², Ribeirão Preto, SP - Brasil
}

\section{Resumo}

A farmacogenética é um dos campos mais promissores da medicina. A conclusão do Projeto Genoma permitiu que esse campo começasse a descobrir fatores complexos modulando a resposta às drogas, e novas tecnologias estão a poucos passos de permitir uma grande expansão da área. As doenças cardiovasculares estão atualmente entre as maiores causas de internações hospitalares e morte, e têm sido alvo de grande parte dos estudos genéticos de doenças complexas. Paralelamente à identificação de marcadores de suscetibilidade à doença, é necessária a investigação de como perfis genéticos diferentes podem alterar respostas aos fármacos atualmente empregados. O sistema biológico que controla a produção endotelial do óxido nítrico tem sido um dos grandes alvos nas respostas farmacológicas aos fármacos usados na terapia de doenças cardiovasculares. Esta revisão tem como objetivo abordar os conhecimentos correntes da interação entre as variações genéticas da eNOS e as respostas farmacológicas aos fármacos empregados no sistema cardiovascular.

\section{Introdução}

Os resultados iniciais do Projeto Genoma foram publicados em $2001^{1}$ e, nestes últimos oito anos, houve um grande avanço no entendimento dos mecanismos moleculares que permeiam as influências genéticas no ser humano. Uma das principais implicações do conhecimento acumulado do genoma humano é a investigação de características genéticas e suas associações a fenômenos até então não explicados.

As características genéticas variáveis (polimorfismos) estudadas se dividem em três grandes grupos: polimorfismos de base única (single nucleotide polymorphisms - SNPs),

\section{Palavras-chave}

Farmacogenética, polimorfismo genético, óxido nítrico, doenças cardiovasculares.

Correspondência: José Eduardo Tanus-Santos •

Av. Bandeirantes 3900 - Monte Alegre - 14090-900 - Ribeirão Preto, SP Brasil

E-mail: tanus@fmrp.usp.br, tanussantos@yahoo.com

Artigo recebido em 08/07/09; revisado recebido em 08/07/09; aceito em 25/01/10. variação no número de repetições de sequências específicas (microssatélites e variable number of tandem repeats) e inserções/deleções de sequências gênicas específicas. Para serem caracterizadas como polimorfismos, essas características devem ter uma frequência estável em pelo menos $1 \%$ na população e, por si só, não serem capazes de causar alguma doença genética. Esses polimorfismos são responsáveis pelas diferenças de características individuais em cada ser humano em nossa população.

Recentemente foi disponibilizada a base de dados do International HapMap Project, que disponibiliza os chamados "tagSNPs" (SNPs que sinalizam a presença de um conjunto específico de alelos em outros polimorfismos próximos). Polimorfismos situados muito próximos entre si tendem a segregar em conjunto dos pais para os filhos, formando haplótipos. A ideia dos "tagSNPs" é genotipar apenas alguns polimorfismos representantes desses haplótipos e inferir os demais por probabilidade. Cada população tem seu conjunto de "tagSNPs", contempladas em grande parte pelo HapMap Project ${ }^{2}$.

Inerentes a todos os tratamentos farmacológicos existem situações de não resposta e toxicidade a doses usuais de fármacos, que não podem ser explicados por comorbidades, situações fisiológicas específicas ou hábitos dos pacientes. A farmacogenética é o campo do conhecimento focado no estudo de interações de polimorfismos genéticos com a farmacologia, tratando tanto de alterações na cinética dos fármacos (absorção, transporte, metabolização e eliminação) como na dinâmica da interação dos fármacos com seus receptores ${ }^{3}$.

A farmacogenética visa detectar previamente características individuais dos pacientes que possam identificá-los como "bons respondedores", ou "maus respondedores", a cada tratamento farmacológico. Milhões de dólares são gastos anualmente em todo o mundo com tratamentos ineficazes (incapazes de tirar o paciente de uma situação de risco), ou com internações por intoxicações farmacológicas; situações que poderiam ser evitadas otimizando a prática da "tentativa e erro" usada na clínica ${ }^{4}$. Os dados são de fato alarmantes: em torno de $7 \%$ do total de internações nos Estados Unidos em 2006 e de 6,5\% no Reino Únido em 2004 foram decorrentes de reações adversas a medicamentos ${ }^{5,6}$. Esses números mostram a grande importância da personalização e racionalização no uso dos medicamentos.

Basicamente, o objetivo maior da farmacogenética é individualizar os tratamentos farmacológicos de maneira racional, direcionada, e, sobretudo, com fortes bases 
científicas para reduzir os efeitos colaterais, a ineficácia terapêutica e seus desdobramentos.

Os avanços na biologia molecular e na bioquímica têm evidenciado o real desafio da farmacogenética: cada via fisiológica tem dezenas de proteínas que interagem entre si; cada proteína, codificada por seu gene (com polimorfismos), pode ter sua transcrição regulada por diversas outras proteínas, cujos genes também têm polimorfismos. Adicionando complexidade a isso, as vias bioquímicas interagem entre si de maneiras complexas (muitas delas ainda desconhecidas), de forma que às vezes o real causador de uma alteração na resposta ao fármaco não é, de forma alguma, óbvio.

Tal complexidade leva a duas correntes genéricas para o estudo da farmacogenética: os estudos baseados em Genome Wide Association Studies (GWAs), e os estudos baseados nas vias fisiológicas e fisiopatológicas dos sistemas biológicos.

Genome Wide Association Studies são estudos grandes que usam técnicas de genotipagem em larga escala, capazes de caracterizar milhares de polimorfismos em todo o genoma em um único experimento. Esse tipo de ensaio permite a geração de resultados sem hipóteses preconcebidas. A ideia por trás da utilização desses estudos na farmacogenética é que a resposta às drogas muitas vezes envolve vias ainda desconhecidas, dificultando a geração de hipóteses a priori ${ }^{7}$. Dessa maneira, tornam-se possíveis estudos clínicos em larga escala sem a necessidade de escolher anteriormente polimorfismos candidatos para associação com determinado fenótipo. GWAs têm um enorme potencial de gerar novas hipóteses, e provavelmente serão capazes de alavancar o desenvolvimento do conhecimento na farmacogenética ${ }^{4} \mathrm{em}$ médio prazo. Contudo, existem sérias limitações nesse tipo do estudo: a necessidade de grandes números de pacientes ou de um grande efeito clínico por parte desses polimorfismos, para que o estudo tenha poder estatístico suficiente para detectar diferenças significantes. GWAs envolvem custos altíssimos, na ordem de milhões de dólares cada ${ }^{8}$; além disso, os grandes esforços de recrutamento de pacientes (os quais não tendem a reduzir de custo como as tecnologias empregadas atualmente ${ }^{4}$ ) dão um indicativo de que GWAS provavelmente se restringirão a grandes centros ricos em financiamentos ${ }^{7}$.

Por definição, GWAs testam apenas variantes comuns dos genes, ignorando completamente variantes raras (que podem, potencialmente, exercer grandes efeitos na resposta a fármacos $)^{9,10}$; além disso, os chips atuais se baseiam nos painéis do HapMap Project para cobrir praticamente a totalidade dos SNPs comuns no genoma, de forma que populações não representadas nesses painéis podem ter erros nas inferências dos haplótipos, em razão de diferenças nos "tagSNPs", não sendo contempladas atualmente nesse tipo de estudo ${ }^{11}$. Existem também limitações técnicas nos estudos por chips de DNA. Para se ter uma ideia, de 83 variantes gênicas com grande importância clínica estudadas por Peters ${ }^{7}$, apenas 45 são contempladas nos painéis do HapMap Project por causa de dificuldades técnicas (impossibilidade de determinar grandes inserções/deleções), mostrando que essa tecnologia pode estar longe de ser a ferramenta definitiva para estudos em farmacogenética.

Outro desafio nos GWAs é a interpretação dos dados; em alguns casos, ocorrem relações que se demonstram nitidamente artefatos por flutuações estatísticas ${ }^{8}$. Até o momento, a maioria dos GWAs se concentrou nos polimorfismos para suscetibilidade a doenças, existindo apenas poucos estudos em farmacogenética, de forma que ainda existe um grande campo para a expansão desse tipo de estudo na resposta diferenciada aos fármacos ${ }^{6}$.

Existe uma outra abordagem, talvez tão eficiente quanto os GWAS, para se estudarem genes que participam de mecanismos fisiológicos já conhecidos ${ }^{8}$. Essa visão baseia-se na sugestão de que se um gene é implicado na causa de uma doença, ou é capaz de influenciar a resposta a um fármaco, provavelmente outros genes na mesma via também podem estar envolvidos ${ }^{8}$. Idealmente, estudos prévios determinam o efeito dos polimorfismos por ensaios moleculares de expressão e/ou atividade enzimática, e os resultados encontrados podem então ser verificados em seres humanos em estudos clínicos ${ }^{10}$. Nesses, a genotipagem é realizada por métodos comuns, e é acompanhada pela análise de marcadores bioquímicos relacionados à proteína afetada, e pela associação com o fenótipo final (doença ou resposta ao tratamento). Essa abordagem genótipo-bioquímica-fenótipo final é de grande valia, pois polimorfismos genéticos podem causar alteração na atividade ou expressão de proteínas. Essas, por sua vez, podem alterar as concentrações plasmáticas de algum produto bioquímico envolvido em um processo fisiopatológico. Dessa forma, tais polimorfismos podem ter grande valor preditivo na clínica médica. Dois exemplos recentes de estudos que podemos citar são: i) o estudo de polimorfismos no gene da aldosterona sintase, com posterior quantificação da concentração de aldosterona circulante, e avaliação da associação com hipertensão resistente ${ }^{12}$; ii) o estudo de polimorfismos na eNOS, quantificação de níveis plasmáticos de nitrito, e investigação da associação com pré-eclampsia $^{13}$. Essa abordagem não necessita de recursos humanos e financeiros tão grandes e, por suas características, possui um grande potencial de gerar resultados mais focados nos problemas clínicos, com aplicabilidade em curto prazo.

Provavelmente, o ideal seria buscar a complementação de GWAs com estudos tradicionais, unindo a grande abrangência dos GWAS com as vantagens de se ter o foco em hipóteses de grande interesse. Essa combinação poderia levar a conclusões mais sólidas, sem negligenciar achados importantes ${ }^{7}$.

Enfim, a farmacogenética é uma ciência nova, que promete grandes impactos na maneira como as doenças serão tratadas. Esta revisão abordará os mecanismos da interação de polimorfismos no gene da sintase endotelial de óxido nítrico com as respostas farmacológicas às drogas cardiovasculares em uso corrente.

\section{Relevância do óxido nítrico para o aparelho cardiovascular}

O endotélio tem um papel importante na regulação da homeostase vascular e na manutenção do tônus vascular, estando envolvido com vias fisiológicas importantes no controle da resistência arterial ao fluxo sanguíneo. Entre diversos mediadores liberados pelo endotélio, o óxido nítrico (NO) exerce papel fundamental na regulação do sistema cardiovascular. Após sua formação pelo endotélio, 


\section{Atualização Clínica}

o NO difunde-se para o músculo liso e interage com o grupo heme da guanilato ciclase solúvel (sGC) tornando-a ativa. Essa enzima sintetiza guanosina monofosfato cíclica (GMPc) a partir da guanosina trifosfato (GTP), levando a um acúmulo de GMPc nessas células. Isso ativa vias de sinalização intracelulares que diminuem o grau de contração do músculo liso vascular, levando ao relaxamento do vaso ${ }^{14}$. Além desses efeitos vasculares, o NO também está envolvido na inibição da agregação e adesão plaquetária.

O NO é formado pelas enzimas NO-sintases (NOS), que catalisam a conversão de L-arginina em L-citrulina e NO. Existem três isoformas dessa enzima: neuronial (nNOS ou NOSI), induzida (iNOS ou NOSII) e endotelial (eNOS ou NOSIII $)^{15}$. A nNOS é encontrada em uma variedade de células que incluem os neurônios e células endoteliais. Tanto a nNOS quanto a eNOS são isoformas constitutivas e são cálcio-dependentes, necessitando do aumento dos níveis de cálcio intracelular e consequente ligação desse com a calmodulina (CaM) para a ativação dessas enzimas. A iNOS não é constitutiva e é expressa em processos inflamatórios.

No sistema cardiovascular, a eNOS é a principal responsável pela síntese de $\mathrm{NO}^{14,16}$. A eNOS localiza-se nas invaginações da membrana plasmática de células endoteliais, denominadas cavéolas. A interação da eNOS com uma proteína chamada caveolina resulta na inatividade da eNOS que se deve, em parte, pela ocupação do sítio de ligação da calmodulina ${ }^{17}$.

A redução da expressão ou da atividade da eNOS pode resultar em menor produção de NO. Diversos estudos têm sugerido que o desequilíbrio na biodisponibilidade do NO exerce papel significativo na disfunção endotelial. Várias doenças estão associadas com disfunção endotelial e redução da biodisponibilidade do NO, entre elas, hipertensão ${ }^{18}$, préeclampsia ${ }^{19}$ e síndrome metabólica ${ }^{20}$.

O estresse oxidativo está envolvido em processos fisiopatológicos de inúmeras doenças cardiovasculares, e existem evidencias que demonstram sua contribuição na disfunção endotelial. As espécies reativas do oxigênio (ROS), como o ânion superóxido, reagem com o NO resultando na formação de peroxinitrito. O aumento na produção de ROS leva, portanto, a uma redução da biodisponibilidade do $\mathrm{NO}^{21}$, podendo favorecer o surgimento de diversas doenças cardiovasculares. O aumento das ROS também pode levar à oxidação do cofator $\mathrm{BH} 4$ da eNOS, levando a um desacoplamento dessa enzima. Dessa forma, a eNOS passa a produzir ânion superóxido em vez de $\mathrm{NO}^{22}$, levando a um ciclo vicioso que aumenta cada vez mais o estresse oxidativo e reduz cada vez mais a disponibilidade de NO.

\section{Relevância do óxido nítrico para as respostas às drogas de ação cardiovascular}

Diversos grupos de drogas usadas no tratamento de doenças cardiovasculares aumentam a biodisponibilidade do NO. Dentre elas, temos os inibidores da enzima conversora de Angiotensina (iECA), antagonistas dos receptores de Angiotensina II (Ang II) e os bloqueadores do canais de cálcio $(\mathrm{BCC})^{23}$. Além desses anti-hipertensivos, diversos estudos têm evidenciado o aumento do NO causados por inibidores da 3-metilglutaril coenzima A redutase (estatinas), provavelmente de forma independente da redução dos níveis de lipídeos ${ }^{24,25}$.

Inibidores da ECA atuam reduzindo as concentrações de Ang II, um potente vasoconstritor endógeno. Paralelamente, ocorre um aumento das concentrações de cininas (cujo efeito predominante é oposto à AngII), e isso contribui para os efeitos cardiovasculares dessas drogas ${ }^{26}$. Os iECA parecem também estimular a expressão e atividade da eNOS ${ }^{27,28}$, provavelmente envolvendo a ligação das cininas aos seus receptores ${ }^{29-31}$. Esses efeitos são inibidos pelo tratamento concomitante com HOE140 , um antagonista do receptor $B_{2}$ de cinina ${ }^{27,32}$.

Os inibidores dos receptores de Ang II são utilizados por inibirem competitivamente a ligação da Ang II ao receptor AT1, atenuando os efeitos vasoconstritores da Ang II. Essas drogas também atuam aumentando a biodisponibilidade de NO, provavelmente pelo aumento da expressão proteína eNOS ou ativação de outros receptores que levam à ativação da eNOS ${ }^{23,33}$.

Os bloqueadores de canais de cálcio (BCC) inibem a entrada de cálcio nas células musculares lisas, e dessa forma levam a uma menor contratibilidade e débito cardíaco. Existem evidências de que essa classe também aumenta a biodisponibilidade do $\mathrm{NO}^{23,34}$. Estudos clínicos evidenciaram significativa melhora da vasodilatação endotélio-dependente em pacientes hipertensos e hipercolesterolêmicos tratados com BCC, mostrando reversão da disfunção endotelial ${ }^{23}$. Provavelmente o aumento do NO é decorrente do aumento da atividade e expressão da eNOS associado a essas drogas ${ }^{35}$.

As estatinas estão entre as drogas mais amplamente usadas e efetivas para prevenção de doenças cardiovasculares ${ }^{36}$, especialmente quando se consideram suas ações sobre as concentrações circulantes de lipoproteínas e seus efeitos cardioprotetores de longo prazo. Alguns dos efeitos pleiotrópicos observados parecem estar associados ao aumento da produção endógena de $\mathrm{NO}^{37,38}$, possivelmente atribuídos ao aumento expressão e atividade da eNOS ${ }^{39,40}$.

A disfunção erétil é uma desordem de origem neuronial e vascular, e vem sendo vista como um fator de risco precoce para outras doenças cardiovasculares ${ }^{41}$. Os inibidores da fosfodiesterase 5 (IPDE-5) são fármacos empregados na disfunção erétil e na hipertensão pulmonar por agirem por meio da inibição da degradação de GMPc, levando a um relaxamento da musculatura lisa dos vasos. No caso da hipertensão pulmonar isso levaria a uma normalização da pressão, e na disfunção erétil facilitaria o intumescimento do pênis decorrente do estímulo neurogênico ${ }^{41}$. Síndromes em que a síntese de NO está comprometida, como diabetes e falência cardíaca, reduzem a eficácia dos IPDE-5 atuais em induzir vasodilatação ${ }^{42,43}$ e alterar a função cardíaca ${ }^{44}$.

\section{Polimorfismos genéticos da eNOS}

O gene da eNOS (localizado na região 7q35-7q36) contém 26 exons, 25 introns e aproximadamente 21 a $22 \mathrm{~Kb}^{45,46}$. Desde a sua caracterização no início dos anos 1990, grande número de sítios polimórficos foi identificado, incluindo VNTRs, repetições de dinucleotídeos (CA)n e SNPs ${ }^{47}$. Vários desses polimorfismos têm sido associados a doenças cardiovasculares, tais como hipertensão, pré-eclampsia, entre outras ${ }^{47-49}$. 
Um dos polimorfismos clinicamente relevantes mais estudados é um SNP na região promotora $\left(\mathrm{T}^{-786} \mathrm{C}\right)$, frequentemente associado ao desenvolvimento de doença coronariana ${ }^{50,51}$. Estudos in vitro indicam que a substituição da timina pela citosina na posição -786 reduz em cerca de 50\% a atividade transcricional ${ }^{51,52}$ (fig. 1A e 1B). Provavelmente, esse efeito ocorra por uma maior ligação da RPA1 (replication protein A1), que atua como uma proteína repressora gênica ${ }^{51}$ em indivíduos com o alelo raro (fig. 1B).

Outro polimorfismo no gene da eNOS amplamente estudado é um VNTR localizado no intron 4 (repetição de $27 \mathrm{pb}$ ). Os alelos mais encontrados apresentam quatro cópias (variante a, mais rara) ou cinco cópias (variante b, mais comum). Os estudos de funcionalidade e de associação desse polimorfismo com eventos cardiovasculares têm demonstrado resultados conflitantes ${ }^{47,53,54}$. Recentemente tem sido proposto que esse polimorfismo regularia a expressão da eNOS pela formação de pequenos RNAs (sirRNA). Células endoteliais contendo cinco cópias apresentam quantidades maiores de sirRNA e menores níveis de mRNA da eNOS do que células contendo quatro cópias $^{55,56}$ (fig. 1C e 1D), o que poderia explicar a associação desse polimorfismo com risco cardiovascular.

Um terceiro polimorfismo, um SNP localizado no exon 7 do gene da eNOS tem sido associado com risco cardiovascular $^{47,57}$. Esse polimorfismo é caracterizado por uma conversão da guanina pela timina na posição 894 do gene, e consequente substituição da glutamina (alelo mais comum) pelo aspartato (alelo mais raro) no resíduo 298 da eNOS (Glu298Asp) $^{45}$. Evidências apontam para menores formações de $\mathrm{NO}$ em indivíduos portadores do alelo Asp, levando a possíveis alterações funcionais ${ }^{58,59}$. Células endoteliais portadoras desse alelo parecem produzir menos NO, por uma diminuição na disponibilidade da eNOS na cavéola dessas células $^{60}$ (fig. 1E e 1F). A localização celular da eNOS e sua ligação à caveolina 1 desempenham papel fundamental para atividade da enzima.

Apesar das evidências discutidas anteriormente, existe certa controvérsia na influência genética da eNOS sobre as doenças cardiovasculares ${ }^{47}$. Uma possível explicação para esses resultados discrepantes pode ser que simples associações clínicas feitas a partir da análise de um único marcador genético (genótipo) com um fenótipo clínico não tenham um poder suficiente para detecção de seus efeitos pequenos. Uma abordagem alternativa seria a análise da combinação de vários marcadores genéticos em paralelo (haplótipo) ${ }^{48,61}$. Por exemplo, Sandrim e cols. ${ }^{62}$ avaliaram a influência dos genótipos/haplótipos da eNOS na elevação da pressão arterial utilizando os três polimorfismos mais comuns ( $\mathrm{T}^{-786} \mathrm{C}$; Glu298Asp e 4b/a). A análise de genótipos

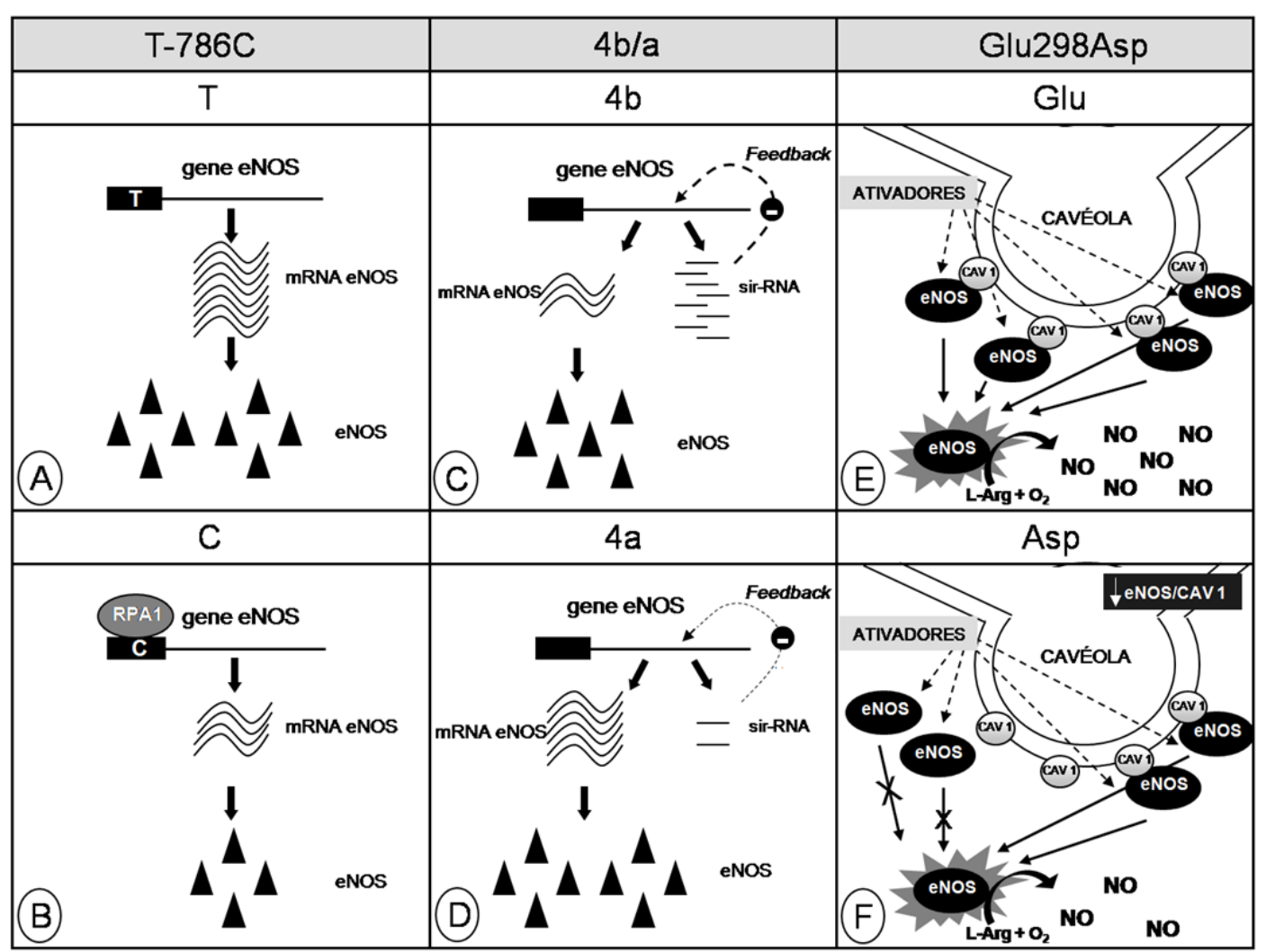

Fig. 1 - Representação dos possiveis mecanismos explicando as associações dos polimorfismos no gene da sintase endotelial do óxido nítrico (eNOS) com variações na sua ação. A e B: polimorfismo na região promotora (T-786C); C e D: polimorfismo no intron 4 (4b/a); E e F: polimorfismo no exon 7 (Glu298Asp). RPA1 (Replication Protein A1), MP (Membrana Plasmática), CAV 1 (Caveolina 1) e sir-RNA (short intronic repeat RNA). Ver detalhes no texto. 


\section{Atualização Clínica}

não evidenciou haver diferenças significativas entre normotensos e hipertensos. Entretanto, a análise haplotípica mostrou claramente a existência de diferenças significativas entre os dois grupos experimentais. Ainda, outros estudos mostraram associação de haplótipos da eNOS com diferentes concentrações circulantes de nitrito, o que sugere que esses haplótipos possam ter implicações funcionais que propiciem risco variado de desenvolvimento de doenças cardiovasculares ${ }^{63,64}$. Essa abordagem haplotípica parece ser mais promissora que a análise de apenas um polimorfismo por vez, especialmente no estudo de doenças complexas.

\section{Estudos de respostas a drogas de ação cardiovascular sendo afetadas por polimorfismos da eNOS}

Estudos têm mostrado relações entre polimorfismos do gene da eNOS com respostas diferenciadas a diversas classes de drogas utilizadas no tratamento de doenças cardiovasculares.

Evidências recentes sugerem uma modulação dos efeitos das estatinas por polimorfismos genéticos da eNOS. Curiosamente, um estudo com células endoteliais em cultura, tratadas com estatinas, mostrou que esses fármacos induzem maiores aumentos dos níveis de mRNA da eNOS em células endoteliais com genótipo CC quando comparadas a células TT para o polimorfismo $\mathrm{T}^{-786} \mathrm{C}^{65}$. Isso se deve, provavelmente, à maior atividade transcricional, aumento da estabilidade do mRNA e diminuição da expressão de RPA $1{ }^{65}$. Confirmando esses achados em culturas de células endoteliais, um estudo clínico mostrou efeitos similares com a atorvastatina ${ }^{66}$. Nesse estudo, demonstrou-se que o tratamento com estatina aumentou a biodisponibilidade do $\mathrm{NO}$ e diminuiu o estresse oxidativo apenas em indivíduos homozigotos CC para o polimorfismo $\mathrm{T}^{-786} \mathrm{C}^{66}$. Note-se que foram estudados indivíduos sadios, fazendo que a droga tivesse muito menos chance de produzir efeitos significativos ${ }^{66}$. Posteriormente, foi observado que os efeitos anti-inflamatórios da atorvastatina são modulados pelo mesmo polimorfismo, levando a uma redução significativa nas concentrações de citocinas inflamatórias (CD40L, VCAM-1, P-selectina e MMP-9) em indivíduos com genótipo CC, mas não nos indivíduos $\mathrm{TT}^{67}$. Estudos funcionais demonstraram subsequentemente que o tratamento de curto prazo com estatinas é capaz de reduzir a fluidez da membrana plasmática de hemácias em indivíduos com genótipo CC, mas não com genótipo TT, durante tratamento com atorvastatina ${ }^{68}$. Juntos, esses achados indicam que a atorvastatina poderia ser mais útil para a prevenção de eventos cardiovasculares em indivíduos com o genótipo CC (cujo risco cardiovascular tem se demonstrado aumentado) do que em indivíduos com genótipo TT.

De qualquer forma, os resultados dos estudos com atorvastatina mencionados devem ser interpretados com cautela, pois não foram avaliados eventos clinicamente relevantes e a simples medida de marcadores bioquímicos pode não ser efetiva para indicar novos usos de drogas. Estudos clínicos enfocando eventos clinicamente relevantes deveriam ser feitos para comprovar os resultados discutidos antes.

Ainda que a maioria dos estudos farmacogenéticos das estatinas relacionados a eNOS centre-se no polimorfismo na região promotora da eNOS, o polimorfimo no íntron
4 também parece modular a resposta às estatinas. Kunnas e cols. ${ }^{69}$ avaliaram a vasodilatação coronariana induzida por adenosina em indivíduos saudáveis após seis meses de tratamento com pravastatina. Os indivíduos portadores do alelo "a" mostraram significativa melhora da vasodilatação quando comparados aos indivíduos com genótipo "bb", possivelmente pelo maior aumento da produção endotelial de NO nos indivíduos com o alelo raro ${ }^{69}$.

Outro polimorfismo no gene da eNOS, Glu298Asp no exon 7, parece modular os efeitos do estradiol na função plaquetária. $\mathrm{O}$ pré-tratamento com $17 \beta$-estradiol aumentou de forma significativa a taxa de agregação plaquetária, acompanhada pela elevação da liberação de íons superóxido pelas plaquetas apenas em indivíduos homozigotos para o alelo Asp298. A liberação de $\mathrm{NO}$ derivado das plaquetas não foi alterada pelos polimorfimos Glu298Asp, $\mathrm{T}^{-786} \mathrm{C}$ e 4b/a após tratamento. Adicionalmente, o efeito do hormônio na função plaquetária não foi afetado pelos polimorfismos na região promotora e ínton $4^{58}$.

Uma das classes de fármacos mais importantes no controle da hipertensão, especialmente no caso de excesso de volume, é a dos diuréticos tiazídicos. A hidroclorotiazida (HCTZ) é seu representante mais utilizado na clínica médica. Foi demonstrado que o polimorfismo Glu298Asp no gene da eNOS é capaz de modular a resposta à HCTZ, de maneira que indivíduos homozigotos para o alelo Glu tem uma redução nos níveis de pressão arterial significativamente maior do que indivíduos carreadores do alelo Asp ${ }^{70}$. Apesar do efeito ser significativo, ele é pequeno, o que evidencia que dificilmente um único polimorfismo será capaz de explicar todo o background genético por trás de variações nas respostas farmacológicas de uma classe de medicamentos.

Outras classes de anti-hipertensivos de grande importância são os betabloqueadores e os inibidores do receptor AT-1 de angiotensina 2. Um estudo usando técnicas análogas aos GWAS mostrou resultados promissores associando polimorfismos menos estudados da eNOS a variações nas respostas farmacológicas de propanolol e irbesartan (betabloqueador e inibidor do receptor AT-1 de angiotensina 2, respectivamente). $\mathrm{O}$ alelo $\mathrm{G}$ do polimorfismo $\mathrm{A}^{2996} \mathrm{G}$ foi associado com uma maior diminuição da pressão arterial decorrente do efeito do betabloqueador atenolol em relação ao alelo $A^{71}$. O alelo $A$ do polimorfismo $\mathrm{G}^{498} \mathrm{~A}$ foi associado a uma melhor resposta tanto ante o betabloqueador quanto ante o irbersartan ${ }^{71}$. A presença dos alelos 2996G e do alelo 498A poderia incorrer em um benefício maior aos portadores desses alelos tratados com esses anti-hipertensivos. Esses resultados, apesar de promissores, ainda devem ser confirmados em estudos com maior número de pacientes, de diferentes populações.

A hipertensão normalmente é tratada com a adição de diferentes classes de anti-hipertensivos até a redução da pressão arterial aos níveis preconizados ${ }^{72}$. Hipertensos resistentes são indivíduos que mantêm seus níveis de pressão arterial acima de 140/90 mmHg apesar do uso de três classes diferentes de anti-hipertensivos, incluindo um diurético. Nesse contexto, foi demonstrada uma tendência não significativa de associação entre o alelo Asp (Glu298Asp) e hipertensão resistente ${ }^{73}$. Estudos posteriores analisando haplótipos da eNOS não confirmaram nenhuma associação com a resistência à terapia anti-hipertensiva ${ }^{62}$. 
A disponibilidade de NO afeta a predisposição à disfunção erétil de forma análoga à hipertensão. A classes dos fármacos utilizados no tratamento da disfunção erétil, os IPE-5, também sofre influência de polimorfismos na sua resposta terapêutica. Como comentado anteriormente, a disponibilidade de NO é um fator determinante para a atividade dos IPDE-5 ${ }^{42}$. Foi demonstrado que homens homozigotos para o alelo Asp (associado à menor produção de NO) obtiveram menor resposta ao sildenafil quando comparados aos indivíduos com genótipo $\mathrm{Glu} / \mathrm{Glu}^{74}$.

\section{Conclusões gerais}

A farmacogenética é uma área da farmacologia bastante recente, e ainda tem muito a crescer. Os resultados do projeto genoma causaram uma promoção e popularização da área, e estamos vivendo atualmente um momento prestes a uma grande expansão, graças aos Genome Wide Association Studies. Apesar disso, a maioria dos estudos ainda se foca nas bases genéticas de doenças complexas, sem grande atenção às respostas farmacológicas. Em relação aos sistemas de produção do óxido nítrico, importantes passos inicias foram dados, e hoje existem fortes indícios que estatinas e estradiol de fato podem sofrer modulação pelo background genético do gene da eNOS em suas funções farmacológicas. Ainda são necessários estudos mais aprofundados em relação aos anti-hipertensivos, e demais medicamentos que atuam sobre o sistema cardiovascular.

É importante frisar que os avanços prometidos pela farmacogenética, infelizmente, ainda estão longe da prática clínica e do paciente.

\section{Perspectivas}

O futuro da medicina caminha cada vez mais para a personalização das terapias, e para o uso racional de tratamentos farmacológicos. A farmacogenética tem um grande espaço nesse cenário hipotético. A ideia, levada a um extremo utópico, seria identificar os polimorfismos genéticos importantes logo ao nascimento, de modo a escolher a priori todos os possíveis futuros tratamentos do indivíduo, adequando as doses ao perfil metabólico predito pelo seu DNA.

A hipertensão, um dos maiores males do mundo moderno, poderia ser tratada de maneira mais eficaz, possivelmente até prevenida, mediante a identificação precoce de marcadores de risco, e do tratamento profilático.

De maneira geral, os avanços genéticos prometem revolucionar a medicina nas próximas décadas; resta avaliar quais das grandes promessas do fim do século XX (terapia gênica, células tronco, farmacogenética) vão realmente dar frutos, e em que proporção.

\section{Agradecimentos}

Agradecemos o apoio financeiro da Fapesp, do CNPq e da Capes.

\section{Potencial Conflito de Interesses}

Declaro não haver conflito de interesses pertinentes.

\section{Fontes de Financiamento}

O presente estudo foi financiado pela FAPESP, CAPES e CNPq.

\section{Vinculação Acadêmica}

Não há vinculação deste estudo a programas de pósgraduação.

\section{Referências}

1. Lander ES, Linton LM, Birren B, Nusbaum C, Zody MC, Baldwin J, et al. Initial sequencing and analysis of the human genome. Nature. 2001; 409 (6822): 860-921.

2. The International HapMap Project. Nature. 2003; 426 (6968): 789-96.

3. Evans WE, McLeod HL. Pharmacogenomics--drug disposition, drug targets, and side effects. N Engl J Med. 2003; 348 (6): 538-49.

4. Gurwitz D, McLeod HL. Genome-wide association studies: powerful tools for improving drug safety and efficacy. Pharmacogenomics. 2009; 10 (2): 157-9.

5. Budnitz DS, Pollock DA, Weidenbach KN, Mendelsohn AB, Schroeder TJ, Annest JL. National surveillance of emergency department visits for outpatient adverse drug events. JAMA. 2006; 296 (15): 1858-66.

6. Pirmohamed M, James S, Meakin S, Green C, Scott AK, Walley TJ, et al. Adverse drug reactions as cause of admission to hospital: prospective analysis of 18820 patients. BMJ. 2004; 329 (7456): 15-9.

7. Peters EJ, McLeod HL. Ability of whole-genome SNP arrays to capture 'must have' pharmacogenomic variants. Pharmacogenomics. 2008; 9 (11): 1573-7.

8. Bader JS. Systems approaches for pharmacogenetics and pharmacogenomics. Pharmacogenomics. 2008; 9 (3): 257-62

9. Topol EJ, Frazer KA. The resequencing imperative. Nat Genet. 2007; 39 (4): $439-40$

10. Dorn GW 2nd, Cresci S. The mechanistic imperative for pharmacogenomics Pharmacogenomics. 2008; 9 (7): 801-3.

11. Marsh S. Pharmacogenetics: global clinical markers. Pharmacogenomics. 2008; 9 (4): 371-3.

12. Lacchini R, Sabha M, Coeli FB, Favero FF, Yugar-Toledo J, Izidoro-Toledo TC, et al. T allele of $-344 \mathrm{C} / \mathrm{T}$ polymorphism in aldosterone synthase gene is not associated with resistant hypertension. Hypertens Res. 2009; 32 (2): 159-62.

13. Sandrim VC, Palei AC, Cavalli RC, Araujo FM, Ramos ES, Duarte G, et al eNOS haplotypes associated with gestational hypertension or preeclampsia. Pharmacogenomics. 2008; 9 (10): 1467-73.

14. Moncada S, Higgs A. The L-arginine-nitric oxide pathway. N Engl J Med. 1993; 329 (27): 2002-12

15. Kiechle FL, Malinski T. Nitric oxide: biochemistry, pathophysiology, and detection. Am J Clin Pathol. 1993; 100 (5): 567-75.

16. Cooke JP, Dzau VJ. Nitric oxide synthase: role in the genesis of vascular disease. Annu Rev Med. 1997; 48: 489-509. 


\section{Atualização Clínica}

17. Dudzinski DM, Igarashi J, Greif D, Michel T. The regulation and pharmacology of endothelial nitric oxide synthase. Annu Rev Pharmacol Toxicol. 2006; 46: 235-76.

18. Perticone F, Ceravolo R, Pujia A, Ventura G, lacopino S, Scozzafava A, et al. Prognostic significance of endothelial dysfunction in hypertensive patients. Circulation. 2001; 104 (2): 191-6.

19. Sandrim VC, Palei AC, Metzger IF, Gomes VA, Cavalli RC, Tanus-Santos JE. Nitric oxide formation is inversely related to serum levels of antiangiogenic factors soluble fms-like tyrosine kinase- 1 and soluble endogline in preeclampsia. Hypertension. 2008; 52 (2): 402-7.

20. Gomes VA, Casella-Filho A, Chagas AC, Tanus-SantosJE. Enhanced concentrations of relevant markers of nitric oxide formation after exercise training in patients with metabolic syndrome. Nitric Oxide. 2008; 19 (4): 345-50.

21. Gryglewski RJ, Palmer RM, Moncada S. Superoxide anion is involved in the breakdown of endothelium-derived vascular relaxing factor. Nature. 1986; 320 (6061): 454-6.

22. Vasquez-Vivar J, Kalyanaraman B, Martasek P, Hogg N, Masters BS, Karoui $\mathrm{H}$, et al. Superoxide generation by endothelial nitric oxide synthase: the influence of cofactors. Proc Natl Acad Sci U S A. 1998; 95 (16): 9220-5.

23. Mason RP, Cockcroft JR. Targeting nitric oxide with drug therapy. J Clin Hypertens (Greenwich). 2006; 8 (12 Suppl 4): 40-52.

24. Liao JK, Laufs U. Pleiotropic effects of statins. Annu Rev Pharmacol Toxicol. 2005; 45: 89-118.

25. Palinski W. New evidence for beneficial effects of statins unrelated to lipid lowering. Arterioscler Thromb Vasc Biol. 2001; 21 (1): 3-5.

26. Sharma JN, Abbas SA. Effect of captopril in the presence of kinin B2 receptor antagonist on duration of survival after prolonged coronary artery ligation in hypertensive rats. Methods Find Exp Clin Pharmacol. 2006; 28 (4): 217-21.

27. Cargnoni A, Comini L, Bernocchi P, Bachetti T, Ceconi C, Curello S, et al. Role of bradykinin and eNOS in the anti-ischaemic effect of trandolapril. Br J Pharmacol. 2001; 133 (1): 145-53.

28. Wiemer G, Linz W, Hatrik S, Scholkens BA, Malinski T. Angiotensin-converting enzyme inhibition alters nitric oxide and superoxide release in normotensive and hypertensive rats. Hypertension. 1997; 30 (5): 1183-90.

29. Zhuo JL, Mendelsohn FA, Ohishi M. Perindopril alters vascular angiotensinconverting enzyme, $\mathrm{AT}(1)$ receptor, and nitric oxide synthase expression in patients with coronary heart disease. Hypertension. 2002; 39 (2 Pt 2): 634-8.

30. Silvestre JS, Bergaya S, Tamarat R, Duriez M, Boulanger CM, Levy BI. Proangiogenic effect of angiotensin-converting enzyme inhibition is mediated by the bradykinin B(2) receptor pathway. Circ Res. 2001; 89 (8): 678-83.

31. Veeravalli KK, Akula A. Involvement of nitric oxide and prostaglandin pathways in the cardioprotective actions of bradykinin in rats with experimental myocardial infarction. Pharmacol Res. 2004; 49 (1): 23-9.

32. Bachetti T, Comini L, Pasini E, Cargnoni A, Curello S, Ferrari R. Ace-inhibition with quinapril modulates the nitric oxide pathway in normotensive rats. J Mol Cell Cardiol. 2001; 33 (3): 395-403.

33. Thai H, Wollmuth J, Goldman S, Gaballa M. Angiotensin subtype 1 rReceptor (AT1) blockade improves vasorelaxation in heart failure by up-regulation of endothelial nitric-oxide synthase via activation of the AT2 receptor. J Pharmacol Exp Ther. 2003; 307 (3): 1171-8.

34. Mason RP, Marche P, Hintze TH. Novel vascular biology of third-generation L-type calcium channel antagonists: ancillary actions of amlodipine. Arterioscler Thromb Vasc Biol. 2003; 23 (12): 2155-63.

35. Kobayashi N, Yanaka H, Tojo A, Kobayashi K, Matsuoka H. Effects of amlodipine on nitric oxide synthase mRNA expression and coronary microcirculation in prolonged nitric oxide blockade-induced hypertensive rats. J Cardiovasc Pharmacol. 1999; 34 (2): 173-81.

36. Zineh I. Pharmacogenetics of response to statins. Curr Atheroscler Rep. 2007; 9 (3): $187-94$

37. Davignon J, Ganz P. Role of endothelial dysfunction in atherosclerosis. Circulation. 2004; 109 (23 Suppl 1): III27-32.

38. Wolfrum S, Jensen KS, Liao JK. Endothelium-dependent effects of statins. Arterioscler Thromb Vasc Biol. 2003; 23 (5): 729-36.
39. Laufs U, La Fata V, Plutzky J, Liao JK. Upregulation of endothelial nitric oxide synthase by HMG CoA reductase inhibitors. Circulation. 1998; 97 (12): 1129-35.

40. Sumi D, Hayashi T, Thakur NK, Jayachandran M, Asai Y, Kano H, et al. A HMGCoA reductase inhibitor possesses a potent anti-atherosclerotic effect other than serum lipid lowering effects--the relevance of endothelial nitric oxide synthase and superoxide anion scavenging action. Atherosclerosis. 2001; 155 (2): 347-57.

41. Jackson G. Treatment of erectile dysfunction in patients with cardiovascular disease: guide to drug selection. Drugs. 2004; 64 (14): 1533-45.

42. Bivalacqua TJ, Usta MF, Champion HC, Leungwattanakij S, Dabisch PA, McNamara DB, et al. Effect of combination endothelial nitric oxide synthase gene therapy and sildenafil on erectile function in diabetic rats. Int J Impot Res. 2004; 16 (1): 21-9.

43. Nagayama T, Zhang M, Hsu S, Takimoto E, Kass DA. Sustained soluble guanylate cyclase stimulation offsets nitric-oxide synthase inhibition to restore acute cardiac modulation by sildenafil. J Pharmacol Exp Ther. 2008; 326 (2): $380-7$

44. Senzaki H, Smith CJ, Juang GJ, Isoda T, Mayer SP, Ohler A, et al. Cardiac phosphodiesterase 5 (cGMP-specific) modulates beta-adrenergic signaling in vivo and is down-regulated in heart failure. FASEB J. 2001; 15 (10): 1718-26.

45. Marsden PA, Heng HH, Scherer SW, Stewart RJ, Hall AV, Shi XM, et al. Structure and chromosomal localization of the human constitutive endothelial nitric oxide synthase gene. J Biol Chem. 1993; 268 (23): 17478-88.

46. Miyahara K, Kawamoto T, Sase K, Yui Y, Toda K, Yang LX, et al. Cloning and structural characterization of the human endothelial nitric-oxide-synthase gene. Eur J Biochem. 1994; 223 (3): 719-26.

47. Cooke GE, Doshi A, Binkley PF. Endothelial nitric oxide synthase gene: prospects for treatment of heart disease. Pharmacogenomics. 2007; 8 (12): 1723-34.

48. Pereira TV, Rudnicki M, Cheung BM, Baum L, Yamada Y, Oliveira PS, et al. Three endothelial nitric oxide (NOS3) gene polymorphisms in hypertensive and normotensive individuals: meta-analysis of 53 studies reveals evidence of publication bias. J Hypertens. 2007; 25 (9): 1763-74.

49. Sandrim VC, Palei AC, Cavalli RC, Araujo FM, Ramos ES, Duarte G, et al. Vascular endothelial growth factor genotypes and haplotypes are associated with pre-eclampsia but not with gestational hypertension. Mol Hum Reprod. 2009: 15 (2): $115-20$

50. Nakayama M, Yasue H, Yoshimura M, Shimasaki Y, Ogawa H, Kugiyama K, et al. T(-786)--> C mutation in the 5'-flanking region of the endothelial nitric oxide synthase gene is associated with myocardial infarction, especially without coronary organic stenosis. Am J Cardiol. 2000; 86 (6): 628-34.

51. Miyamoto Y, Saito Y, Nakayama M, Shimasaki Y, Yoshimura T, Yoshimura M, et al. Replication protein A1 reduces transcription of the endothelial nitric oxide synthase gene containing a $-786 \mathrm{~T}-->\mathrm{C}$ mutation associated with coronary spastic angina. Hum Mol Genet. 2000; 9 (18): 2629-37.

52. Nakayama M, Yasue H, Yoshimura M, Shimasaki Y, Kugiyama K, Ogawa H, et al. T-786-->C mutation in the $5^{\prime}$-flanking region of the endothelial nitric oxide synthase gene is associated with coronary spasm. Circulation. 1999; 99 (22): 2864-70

53. Wang XL, Mahaney MC, Sim AS, Wang J, Wang J, Blangero J, et al. Genetic contribution of the endothelial constitutive nitric oxide synthase gene to plasma nitric oxide levels. Arterioscler Thromb Vasc Biol. 1997; 17 (11): 3147-53.

54. Tsukada T, Yokoyama K, Arai T, Takemoto F, Hara S, Yamada A, et al. Evidence of association of the ecNOS gene polymorphism with plasma NO metabolite levels in humans. Biochem Biophys Res Commun. 1998; 245 (1): 190-3.

55. Zhang MX, Zhang C, Shen YH, Wang J, Li XN, Chen L, etal. Effect of 27nt small RNA on endothelial nitric-oxide synthase expression. Mol Biol Cell. 2008;19 (9): 3997-4005

56. Zhang MX, Zhang C, Shen YH, Wang J, Li XN, Zhang Y, et al. Biogenesis of short intronic repeat 27-nucleotide small RNA from endothelial nitric-oxide synthase gene. J Biol Chem. 2008; 283 (21): 14685-93.

57. Casas JP, Bautista LE, Humphries SE, Hingorani AD. Endothelial nitric oxide synthase genotype and ischemic heart disease: meta-analysis of 26 studies involving 23028 subjects. Circulation. 2004; 109 (11): 1359-65.

58. Tanus-Santos JE, Desai M, Deak LR, Pezzullo JC, Abernethy DR, Flockhart DA, et al. Effects of endothelial nitric oxide synthase gene polymorphisms 
on platelet function, nitric oxide release, and interactions with estradiol. Pharmacogenetics. 2002; 12 (5): 407-13.

59. Godfrey V, Chan SL, Cassidy A, Butler R, Choy A, Fardon T, et al. The functional consequence of the Glu298Asp polymorphism of the endothelial nitric oxide synthase gene in young healthy volunteers. Cardiovasc Drug Rev. 2007; 25 (3): 280-8.

60. Joshi MS, Mineo C, Shaul PW, Bauer JA. Biochemical consequences of the NOS3 Glu298Asp variation in human endothelium: altered caveolar localization and impaired response to shear. Faseb J. 2007; 21 (11): 2655-63.

61. Crawford DC, Nickerson DA. Definition and clinical importance of haplotypes. Annu Rev Med. 2005; 56:303-20.

62. Sandrim VC, Yugar-Toledo JC, Desta Z, Flockhart DA, Moreno H Jr, TanusSantos JE. Endothelial nitric oxide synthase haplotypes are related to blood pressure elevation, but not to resistance to antihypertensive drug therapy. J Hypertens. 2006; 24 (12): 2393-7.

63. Metzger IF, Sertorio JT, Tanus-Santos JE. Modulation of nitric oxide formation by endothelial nitric oxide synthase gene haplotypes. Free Radic Biol Med. 2007; 43 (6): 987-92.

64. Metzger IF, Souza-Costa DC, Marroni AS, Nagassaki S, Desta Z, Flockhart DA, et al. Endothelial nitric oxide synthase gene haplotypes associated with circulating concentrations of nitric oxide products in healthy men. Pharmacogenet Genomics. 2005; 15 (8): 565-70.

65. Abe K, Nakayama M, Yoshimura M, Nakamura S, Ito T, Yamamuro M, et al. Increase in the transcriptional activity of the endothelial nitric oxide synthase gene with fluvastatin: a relation with the $-786 \mathrm{~T}>$ C polymorphism. Pharmacogenet Genomics. 2005; 15 (5): 329-36.

66. Nagassaki S, Sertorio JT, Metzger IF, Bem AF, Rocha JB, Tanus-Santos JE. eNOS gene T-786C polymorphism modulates atorvastatin-induced increase in blood nitrite. Free Radic Biol Med. 2006; 41 (7): 1044-9.
67. Souza-Costa DC, Sandrim VC, Lopes LF, Gerlach RF, Rego EM, Tanus-Santos JE. Anti-inflammatory effects of atorvastatin: modulation by the T-786C polymorphism in the endothelial nitric oxide synthase gene. Atherosclerosis. 2007; 193 (2): 438-44.

68. Nagassaki S, Herculano RD, Graeff CF, Tanus-Santos JE. eNOS T-786C polymorphism affects atorvastatin-induced changes in erythrocyte membrane fluidity. Eur J Clin Pharmacol. 2009; 65 (4): 385-92.

69. Kunnas TA, Lehtimaki T, Laaksonen R, Ilveskoski E, Janatuinen T, Vesalainen R, et al. Endothelial nitric oxide synthase genotype modulates the improvement of coronary blood flow by pravastatin: a placebo-controlled PET study. J Mol Med. 2002; 80 (12): 802-7.

70. Turner ST, Chapman AB, Schwartz GL, Boerwinkle E. Effects of endothelia nitric oxide synthase, alpha-adducin, and other candidate gene polymorphisms on blood pressure response to hydrochlorothiazide. Am J Hypertens. 2003; 16 (10): 834-9.

71. Liljedahl U, Karlsson J, Melhus H, Kurland L, Lindersson M, Kahan T, et al. A microarray minisequencing system for pharmacogenetic profiling of antihypertensive drug response. Pharmacogenetics. 2003; 13 (1): 7-17.

72. Chobanian AV, Bakris GL, Black HR, Cushman WC, Green LA, Izzo JL Jr et al. The Seventh Report of the Joint National Committee on Prevention, Detection, Evaluation, and Treatment of High Blood Pressure: the JNC 7 report. JAMA. 2003; 289 (19): 2560-72.

73. Jachymova M, Horky K, Bultas J, Kozich V, Jindra A, Peleska J, et al. Association of the Glu298Asp polymorphism in the endothelial nitric oxide synthase gene with essential hypertension resistant to conventional therapy. Biochem Biophys Res Commun. 2001; 284 (2): 426-30.

74. Eisenhardt A, Sperling H, Hauck E, Porst H, Stief C, Rubben H, et al. ACE gene I/D and NOS3 G894T polymorphisms and response to sildenafil in men with erectile dysfunction. Urology. 2003; 62 (1): 152-7. 